\title{
Untreated Childhood Low Grade Astrocytic Tumor
}

National Cancer Institute

\section{Source}

National Cancer Institute. Untreated Childhood Low Grade Astrocytic Tumor. NCI

Thesaurus. Code C114961.

A finding of childhood low grade astrocytic tumor that has not been treated. 